\title{
The Impact of Dispersion Slope on Fiber Nonlinearity in Ultra-Wideband Optical Communication System
}

\author{
Abdallah A. I. Ali (1), F. Ferreira ${ }^{(1)}$, M. Al-Khateeb(1), D. Charlton ${ }^{(2)}$, C. Laperle(2) , A. D. Ellis ${ }^{(1)}$
}

(1) Aston Institute of Photonic Technologies, Aston University, Birmingham, UK, aliaai@aston.ac.uk

(2) Ciena Corporation, 383 Terry Fox Drive, Ottawa, ON, K2K OL1, Canada.

\begin{abstract}
We investigate the impact of dispersion slope induced phase-matching on nonlinear noise for wide-bandwidth signals. To ignore the third-order dispersion, its product with 6-times the bandwidth should be less than the second-order dispersion, as for wavelengths from 1360-1570nm in SSMF.
\end{abstract}

\section{Introduction}

To meet future high capacity demand, multiple telecom optical wavelength bands are now being used in commercial systems ${ }^{1}$. To-date, the increased nonlinear distortion arising from the wider bandwidth signals has been well modelled by assuming constant fiber parameters ${ }^{2}$. Even with the known vulnerabilities of optical phase conjugation to dispersion-power asymmetry, the impact of dispersion slope has yet to be observed for coherent transmission systems ${ }^{3}$. However, it is evident that as signal bandwidths continue to grow, such wavelength independent parameter assumptions may become inaccurate and more detailed understanding of bandwidth dependent effects will become increasingly important.

In this paper, we analyse the effect of dispersion slope on the nonlinear performance of wideband signals, proposing a normalised parameter beyond which dispersion slope must be taken into account, and verifying our analysis by experimentally measuring nonlinear noise (NLN) and system performance over a dispersion shifted fibre (DSF) link, which scales the effects to narrower bandwidths. In particular, we show that the effect of dispersion slope is significant if the product of $2 \pi$ times signal bandwidth and third order dispersion coefficient exceeds the second order dispersion coefficient $\left(\beta_{3} \Delta \omega>\beta_{2}\right)$, and may influence system performance before the dispersion zero is hit.

\section{FWM with Dispersion Slope}

The four wave mixing (FWM) power ${ }^{4}$ resulting from the interaction of three signals at frequencies $f_{1}, f_{2}$ and $f_{3}$ with powers $P_{1}, P_{2}$ and $P_{3}$ in single span of single mode fiber (SMF) is given by,

$$
\begin{aligned}
P_{F W M} & =\frac{D_{g}^{2} \gamma^{2} P_{1} P_{2} P_{3}}{9\left(\alpha^{2}+\Delta \beta^{2}\right)}\left(\left(1-e^{-\alpha L}\right)^{2}\right. \\
& \left.+4 e^{-\alpha L} \sin ^{2}(\Delta \beta L / 2)\right)
\end{aligned}
$$



Fig. 1: FWM efficiency as a function of frequency separation between the three lasers normalized to the ratio of dispersion slope to the dispersion. The red hexagon represent the NLN boundaries affecting the signal with the same normalization

where $D_{g}$ is the degeneracy factor. $L, \alpha, \gamma$ are the fiber length, attenuation, non-linear coefficient respectively and $\Delta \beta$ is the phase mismatch. For SMF, it may be approximated ${ }^{5}$ by considering only the second $\beta_{2}$ and the third order dispersion $\beta_{3}$ (approximation 2) as,

$$
\begin{aligned}
\Delta \beta & =-(2 \pi)^{2} \beta_{2}\left(f_{1}-f_{3}\right)\left(f_{2}-f_{3}\right)(1 \\
& \left.+\pi \frac{\beta 3}{\beta 2}\left(\left(f_{1}-f_{3}\right)+\left(f_{2}-f_{3}\right)\right)\right)
\end{aligned}
$$

Conventionally Eq.(2) is further approximated by considering $\beta_{3}$ to be zero but with $\beta_{2}$ evaluated at the centre of the signal band (approximation 1). From Eq.(2), there are three regimes of strong phase matching $(\Delta \beta \approx 0)$, where two of the frequencies are close to each other $\left(f_{1} \approx f_{3}\right.$ and $f_{2} \approx$ $\left.f_{3}\right)$ and a third when $\pi \frac{\beta 3}{\beta 2}\left(\left(f_{1}-f_{3}\right)+\left(f_{2}-f_{3}\right)\right)=-1$.

By normalizing the frequency separation to the ratio of dispersion slope to dispersion, Fig.(1) shows the FWM efficiency in logarithmic scale as a function of the frequency septation. The figure plotted for $80 \mathrm{~km}$ of DSF with dispersion $\left(\beta_{2}\right)-0.54$ $\mathrm{ps}^{2} / \mathrm{km}$, third order dispersion $\left(\beta_{3}\right) 0.131 \mathrm{ps}^{3} / \mathrm{km}$ and attenuation $0.26 \mathrm{~dB} / \mathrm{km}$ at $193.6 \mathrm{THz}$. The blue regions represent low FWM efficiency due 
to weak phase matching. The yellow areas represent the three areas of strong phase matching. The dispersion slope has three key effects. Firstly, the appearance of an additional strongly phase matched region (diagonal yellow peak), secondly, the modification of phase matching in the region bounded by the three strongly phase matched regions, and a slight change in the rate of decay in the weakly phase matched area. These general features remain irrespective of the fibre type, although the width of the strongly phase matched regions (yellow) decreases with $\beta_{2}$. The red polygon bounds the region where signals may interact to produce mixing products at the centre of the signal band, and scales with fibre dispersion and bandwidth. Additional strongly phase matched mixing products are clearly to be expected if the polygon includes all three phase matching peaks. For standard SMF, this occurs with bandwidths around $20 \mathrm{THz}$, assuming a centre frequency of $193.6 \mathrm{THz}(30 \mathrm{THz}$ assuming the centre frequency shifts to higher frequency with increasing bandwidth) and for Non-zero dispersion shifted fiber (NZ-DSF) (dispersion $\sim-5$ $\left.\mathrm{ps}^{2} / \mathrm{km}\right) \sim 5.9 \mathrm{THz}$. However, an increase in nonlinear noise (NLN) may occur before these bandwidths, and should be calculated by integrating Eq.(1) taking into account dispersion slope.

\section{Experimental Results and Discussion}

We have employed DSF to experimentally verify the accuracy of the normalized analytical model. We first verified the FWM efficiency (Fig.(1)) by combining two tunable continuous wave lasers using polarization maintaining coupler and launching at $15 \mathrm{dBm}$ into a $6.4 \mathrm{~km}$ length of DSF with the same parameters as used for Fig.(1), recording the idler power as a function of frequency separation with a high resolution (150 $\mathrm{MHz}$ ) optical spectrum analyser (Fig. (2)).

Two different frequency ranges were selected to illustrate the changes in efficiency due to the emergence of dispersion slope effects. For the lower curves (red and light blue), one laser was fixed at 194.6THz, whilst the other was swept up to $195.4 \mathrm{THz}$. The effect of dispersion slope is quite small and only appears as slight asymmetry between the weakly phase matched idler powers for positive and negative detuning. With the fixed laser tuned to $193.6 \mathrm{THz}$, closer to the dispersion zero (green and dark blue), the impact of dispersion slope becomes significant and appears as an enhancement in the FWM efficiency between 0.2

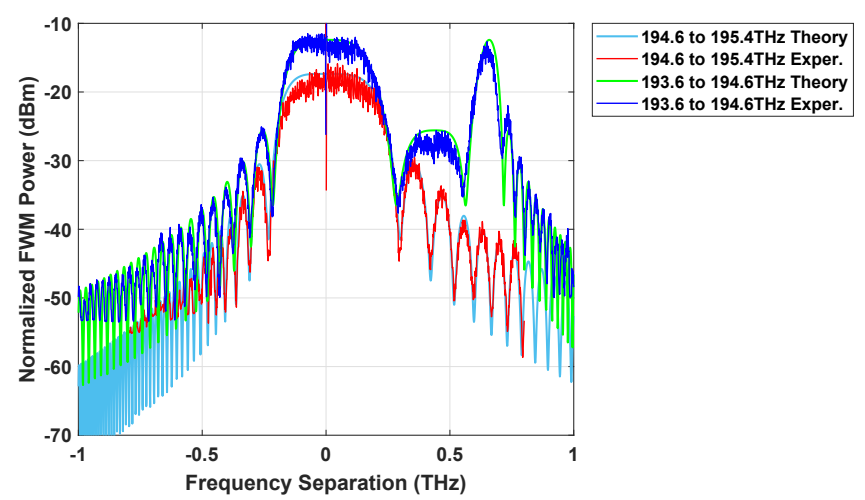

Fig. 2: FWM efficiency as a function of frequency separation between two lasers showing experimental results (red and blue) and theory (green and light blue) with differnt laser frequencies. Blue and green curves shifted up by $5 \mathrm{~dB}$.

and $0.5 \mathrm{THz}$ with an extra peak around $0.65 \mathrm{THz}$ separation. Excellent matching with the theoretical predictions may be observed.

Next, we measured the NLN power spectral density (PSD) using the spectral notch method ${ }^{6}$ as shown in Fig.(3). An amplified spontaneous emission (ASE) was further amplified by a second EDFA and shaped using two wavelength selective switches (WSS) to give a flat spectrum representing Nyquist wave division multiplexing channels with a launched PSD of $0.75 \mu \mathrm{W} / \mathrm{GHz}$ and a $10 \mathrm{GHz}$ notch in the middle of the spectrum representing the location of the channel under test. Three different central notch locations were measured (193.275, 293.75 and $194.25 \mathrm{THz}$ ), representing high, intermediate, and low dispersion spectral regions of the fibre respectively. Figure (4) shows the measured and analytically predicted (taking into account residual background ASE from the WSS cascade) NLN PSD. At small bandwidth, all notches have similar NLN PSDs and for the notch centred at 193.275THz (high dispersion case), the NLN follows the expected logarithmic increase with bandwidth. However, for lower dispersions (notches at 193.75THz and 194.25THz), this logarithmic dependence only holds up to a certain bandwidth, beyond which the dispersion slope takes effect. At moderate dispersion (193.75THz), the additional phase matching condition results in an increase in NLN, reminiscent of the step changes observed in fewmode fibre when additional modes become phase matched $^{7}$. However, for the lowest dispersion $(194.25 \mathrm{THz})$, the effect is to reduce the rate of growth, as widely separated signals are less well phase matched thanks to the dispersion slope. Analytical predictions according to the two approximations considered here, $\beta_{3}=0$ (dashed) and $\beta_{3} \neq 0$ (solid), are also shown where $\beta_{2}$ 




Fig. 3: Experimental setup for NLN and BER measurements. Inset: NLN spectrum at 193.275THz (left) and at 194.25THz (right)

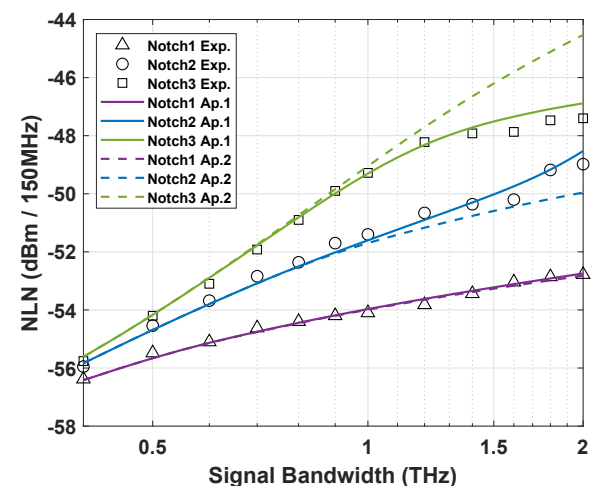

Fig. 4: NLN as a function of the emulated signal bandwidth for Experiment (Markers) and Theory (Lines) using with

(solid) and without (dashed) dispersion slope.

Notch1:193.275THz, Notch2: 193.75THz, Notch3:

194.25THz, Exp.: Experiment and Ap.: Approximation

is adjusted to the correct value at the centre of each band. Despite adjustment of $\beta_{2}$ to the centre of the band (approximation 2), inclusion of dispersion slope is necessary for wider bandwidths $\left(\Delta f>\frac{\beta_{2}}{\pi \beta_{3}}\right)$.



Fig. 5: BER as a function of the transmission bandwidth for Experiment (Markers) and Theory (Lines) with (Solid) and without (Dashed) dispersion slope for QPSK (Triangle and

Diamond) and 16QAM (Circle and Square) signals at 193.3THz (Blue and Orange lines) and at 194.25THz (Green and Purple lines) respectively.

Finally, we verified the impact of the NLN by measuring the bit error rate (BER) for 35Gbaud PM-QPSK and PM-16QAM signals (Fig. (3)). The central notch was increased from $10 \mathrm{GHz}$ to $40 \mathrm{GHz}$ using the WSS and a modulated signal, generated and received using a commercial Ciena WaveLogic 3 transceiver, was inserted in the notch using $3 \mathrm{~dB}$ coupler. An EDFA was used after the transmitter to equalise the power spectral densities. Figure (5) shows the measured BERs as a function of the transmission bandwidth and curve fits based on calculating NLN using approximations 1 and 2 and the conventional dependence of BER on SNR. The results confirm that in addition to predicting FWM efficiencies ${ }^{4,5}$, Eq. (1) may be effectively integrated to estimate NLN and system performance in the presence of significant effects of dispersion slope.

\section{Conclusions}

We have described the impact of dispersion slope on NLN generation, experimentally verifying the predictions using DSF. The analytical predictions suggest that dispersion slope may be safely neglected until $B W>\frac{\beta_{2}}{6 \beta 3}$, approximately $20 \mathrm{THz}$ for fiber with dispersion $\sim-20 \mathrm{ps}^{2} / \mathrm{km}$.

\section{Acknowledgements}

This work has been partially supported by the EPSRC (EP/L000091/1-PEACE) and the European Union (654809-HSPACE). The data underlying this publication can be found at https://doi.org/10.17036/researchdata.aston.ac.uk.00000353

\section{References}

[1] J.-X. Cai et. al., "49.3 Tb/s transmission over $9100 \mathrm{~km}$ using C+ L EDFA and $54 \mathrm{tb} / \mathrm{s}$ transmission over $9150 \mathrm{~km}$ using hybrid-raman EDFA," J. Lightwave Technol., vol. 33, no. 13, p. 2724, 2015.

[2] G. Saavedra et al., "Experimental investigation of nonlinear signal distortions in ultra-wideband transmission systems," in Proc. OFC, WG1.1, Los Angeles, 2017.

[3] F. Da Ros et al., "Impact of signal-conjugate wavelength shift on optical phase conjugation-based transmission of qam signals," in Proc. ECOC, Gothenburg, 2017.

[4] D. Cleland et al., "Precise modelling of four wave mixing products over $400 \mathrm{~km}$ of step-index fibre," Electron Lett., vol. 28 , no. 12 , p. $1171,1992$.

[5] K. Inoue, "Four-wave mixing in an optical fiber in the zero-dispersion wavelength region," J. Lightwave Technol., vol. 10, no. 11, p. 1553, 1992.

[6] A. Ellis et. al, "Expressions for the nonlinear transmission performance of multi-mode optical fiber," Opt. Express, vol. 21, no. 19, p. 22834, 2013.

[7] F. M. Ferreira et al., "Advantages of strong mode coupling for suppression of nonlinear distortion in few-mode fibers," in Proc. OFC, Tu2E.3, Anaheim, 2016. 